\title{
Underwater Acoustic Source Localisation Among Blind and Sighted Scuba Divers Comparative study
}

"Jacopo Cambi, Ludovica Livi, Walter Livi

$$
\text { توطين المصدر الصوني تحت الماء بين الغواصين المكفوفين والمبصرين }
$$

جاكويو كامبي، لودوفيكا ليفي، والتر ليفي

ABSTRACT: Objectives: Many blind individuals demonstrate enhanced auditory spatial discrimination or localisation of sound sources in comparison to sighted subjects. However, this hypothesis has not yet been confirmed with regards to underwater spatial localisation. This study therefore aimed to investigate underwater acoustic source localisation among blind and sighted scuba divers. Methods: This study took place between February and June 2015 in Elba, Italy, and involved two experimental groups of divers with either acquired $(\mathrm{n}=20)$ or congenital $(n=10)$ blindness and a control group of 30 sighted divers. Each subject took part in five attempts at an under-water acoustic source localisation task, in which the divers were requested to swim to the source of a sound originating from one of 24 potential locations. The control group had their sight obscured during the task. Results: The congenitally blind divers demonstrated significantly better underwater sound localisation compared to the control group or those with acquired blindness $(P=0.0007)$. In addition, there was a significant correlation between years of blindness and underwater sound localisation $(P<0.0001)$. Conclusion: Congenital blindness was found to positively affect the ability of a diver to recognise the source of a sound in an underwater environment. As the correct localisation of sounds underwater may help individuals to avoid imminent danger, divers should perform sound localisation tests during training sessions.

Keywords: Blindness; Auditory Perception; Sound Localization; Spatial Processing; Diving.

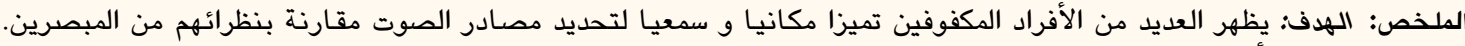

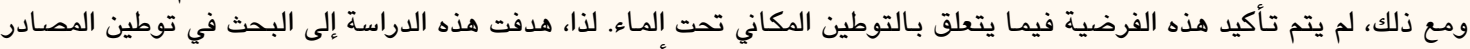

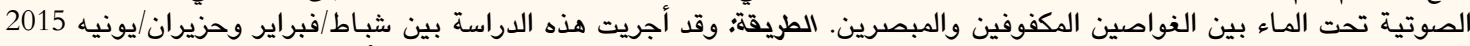

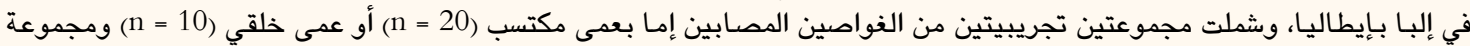

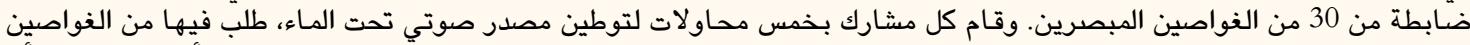

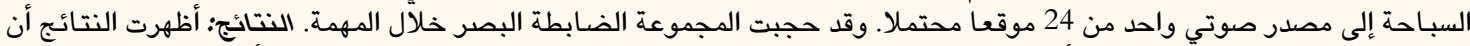

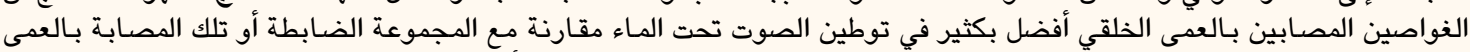

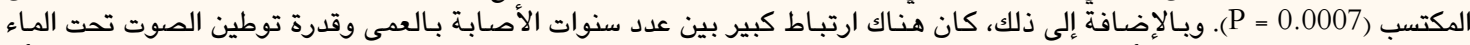

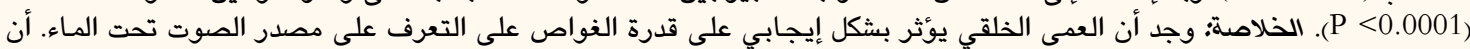

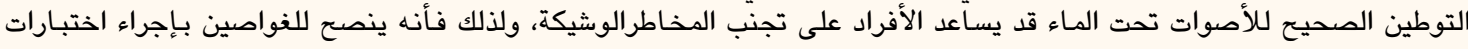
توطين الصوت خلال الدورات التدريبية.

الكلمات المفتاحية: العمى؛ الإدراك السمعي؛ توطين الصوت؛ المعالجة المكانية؛ الغوص.

\section{AdVANCES IN KNOWLEDGE}

To the best of the authors' knowledge, this study is the first to investigate the sound recognition capabilities of both blind and sighted subjects in an underwater environment.

The findings of this study indicated that subjects who were congenitally blind demonstrated superior spatial discrimination of an underwater sound source.

Among individuals who are blind, sensory substitution in the cortex may explain enhanced localisation of an underwater sound source, which is an extremely difficult task for a person who is sighted.

\section{Application to Patient Care}

The findings of this study indicated that congenitally blind subjects were significantly more able to locate underwater sound sources. As such, healthcare practitioners should encourage both sighted divers and those with acquired blindness to undertake additional sound localisation tests during training sessions. 
S OUND WAVES TRAVEL THROUGH THE AIR AT A speed of approximately $300 \mathrm{~m} / \mathrm{second}$; however, since water is denser than air, sound waves

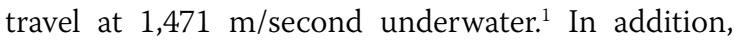
variations in salinity, temperature and pressure (i.e. depth) also influence the propagation of sound underwater. ${ }^{1}$ The human ear functions relatively poorly underwater because the external ear canal fills with water, the eardrum moves less and the resonant frequency shifts to the bass tones. In such circumstances, most individuals hear via bone conduction and scuba divers consequently are more able to perceive low-frequency sounds than high-frequency sounds; however, the water has an overall sound damping effect. $^{2}$ Another peculiarity of underwater hearing is the difficulty in establishing the precise location of a source of sound; this therefore has important safety implications for scuba divers in terms of hazard perception.

The ability of the brain to learn or relearn to localise sounds does not necessitate visuomotor feedback as the neural paths assigned to spatial hearing can be set up in a vision-independent manner. ${ }^{3}$ Previous research on sound localisation among people who are blind has been based on the theory of crossmodal compensatory plasticity in which increased cortical activity has been hypothesised to compensate or hypercompensate for the loss of visual calibration in spatial hearing by employing non-visual sensory signals. ${ }^{4}$ Using neuroimaging techniques, several studies have suggested that posterior visual areas in subjects who are blind may be active during the performance of non-visual tasks such as auditory localisation., ${ }^{5,6}$

Nevertheless, while some studies have reported that blind individuals show better auditory spatial discrimination or localisation of sound sources than sighted subjects, other studies have failed to demonstrate this gain. ${ }^{7-10}$ Recently, Finocchietti et al. reported that localisation of sound in the mid-sagittal plane was poorer among blind individuals than sighted controls, indicating a clear deficit in encoding the sound motion in the lower hemisphere of the spherical coordinate system; however, the researchers confirmed that no such deficit was observed among congenitally blind subjects. ${ }^{11}$ Voss et al. confirmed that individuals who are blind demonstrated supernormal abilities in the recognition of sound sources both nearby and at a distance. ${ }^{12}$ However, several other researchers have reported inferior performances during experimental tasks of spatial sound recognition among subjects who were blind compared to those who were sighted..$^{13,14}$

To the best of the authors' knowledge, the localisation of spatial sounds underwater has never before been evaluated among both blind and sighted subjects. This is significant as, in water, sounds can arise from any point in a three-dimensional setting, including the vertical as well as horizontal planes. This study therefore aimed to evaluate underwater sound recognition among both blind and sighted scuba divers as well as to evaluate differences in sound recognition between subjects with acquired blindness and those with congenital blindness.

\section{Methods}

This study took place between February and June 2015 in Elba, Italy. A total of 60 subjects were recruited to participate in the study, of which 30 participants had either acquired $(n=20)$ or congenital ( $\mathrm{n}=10)$ visual impairment and 30 had no visual impairment. Subjects were considered congenitally blind if their visual acuity was below 1/300 within the first year of life, indicating that they were unable to detect hand movements at a distance of $1 \mathrm{~m}$ from their better eye. All participants had been scuba diving for at least a year before the beginning of the study; the sighted divers had a minimum of an advanced open water diver qualification and at least 20 underwater dives in the preceding two years. The blind subjects were recruited with the aid of blind scuba diver partnerships. An audiological and motor function examination was performed on all subjects, including otoscopy, pure tone audiometry, tympanometry and tubaric function tests. All of the subjects had normal hearing levels at $\leq 20$ decibels ( $d B$ ) and no history of hearing disorders.

Sound localisation tasks were performed in the Secca della Torre, a bay with a rocky seabed located in Marciana Marina, Elba. The tasks took place in an underwater test area of $10 \mathrm{~m} \times 10 \mathrm{~m}$ in which the average depth was $22 \mathrm{~m}$ and the maximum depth was $38 \mathrm{~m}$. Each subject entered the water and moved to the centre of the test area at a depth of approximately $5 \mathrm{~m}$. Subsequently, a sound was produced approximately $5 \mathrm{~m}$ away from the diver by rhythmically hitting a metal screwdriver against the side of a diving tank at a frequency of $1 \mathrm{~Hz}$ and a rate of 60 times per minute. The estimated sound pressure level was $90 \mathrm{~dB}$ with a frequency band range of $2-8 \mathrm{KHz}$.

The sound originated from one of 24 possible locations with each location varying by $45^{\circ}$ on three horizontal planes (either on the surface, at a depth of $5 \mathrm{~m}$ or at a depth of $10 \mathrm{~m}$ ). Each plane had eight possible origin points of the sound. The sound source was determined randomly by throwing a 24 -sided polyhedral dice [Figure 1]. Each subject repeated the task five times. Some of the individuals in the congenital blindness group had residual light perception and were 


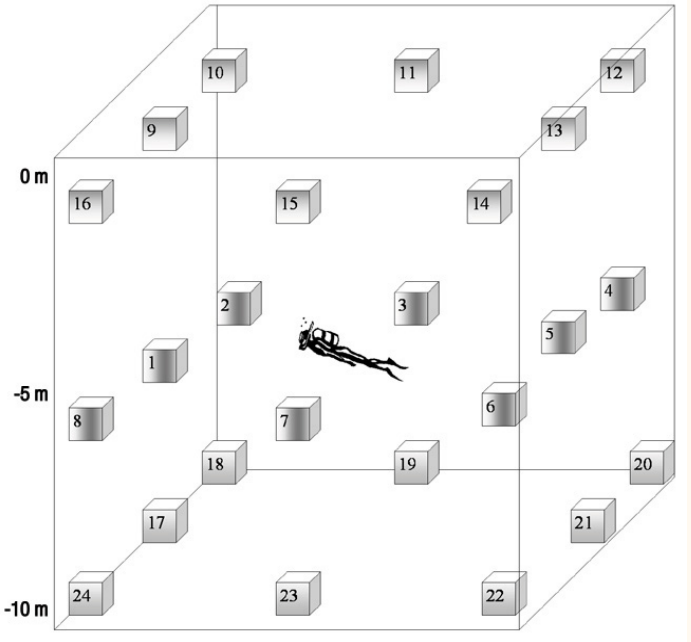

Figure 1: Diagram showing the experimental set-up of an underwater sound localisation task for blind and sighted scuba divers. Each task took place in an underwater test area of $10 \mathrm{~m} \times 10 \mathrm{~m}$ with the diver located in the middle at a depth of $5 \mathrm{~m}$. Subsequently, sounds originated from one of 24 potential sources on three horizontal planes, with the diver required to reach the sound source within one minute in as direct a route as possible.

therefore required to perform the test with their eyes closed. Subjects in the control group were requested to perform the tests with closed eyes while wearing a diving mask which obscured their vision.

Completion of the sound localisation task was scored as follows. Subjects were assigned one point if they reached the sound source in less than a minute without changing course. Half a point was awarded if they reached a position within $45^{\circ}$ of the sound source on the horizontal plane and then corrected their route (e.g. if the sound source was at position nine and the diver initially swam towards positions 16 or 10 ) or if they reached a position deeper or shallower than the sound source and then corrected their route (e.g. if the sound source was at position nine and the diver initially swam towards position one). The participants were assigned zero points if they did not reach the sound source within one minute or swam more than $45^{\circ}$ away from the sound source. The total number of points for the five task attempts was then summed up for each diver to constitute the total task score.

Data were analysed using the Statistical Package for the Social Sciences (SPSS), Version 16.0 (IBM Corp., Armonk, New York, USA). The participants were divided into three groups for the purposes of the statistical analysis: the acquired blindness group, the congenital blindness group and the control group of sighted subjects. Percentages of exact localisation of the sound source were computed independently for each subject and each group. Comparisons between groups were assessed using either a t-test, analysis of variance or Spearman's rank-correlation coefficient analysis, as appropriate. A $P$ value of $<0.0500$ was considered statistically significant.

This study was performed in accordance with the ethical guidelines of the Declaration of Helsinki. Informed written consent was obtained from all of the participants prior to their inclusion in the study.

\section{Results}

Among the 60 divers included in the study, 20 were female and 40 were male. The mean age was $36.9 \pm 10.1$ years. The mean number of years of diving experience was $5.0 \pm 1.4$, while the mean

Table 1: Demographic and clinical characteristics and test scores of blind and sighted scuba divers participating in an underwater sound localisation task $(\mathrm{N}=60)$

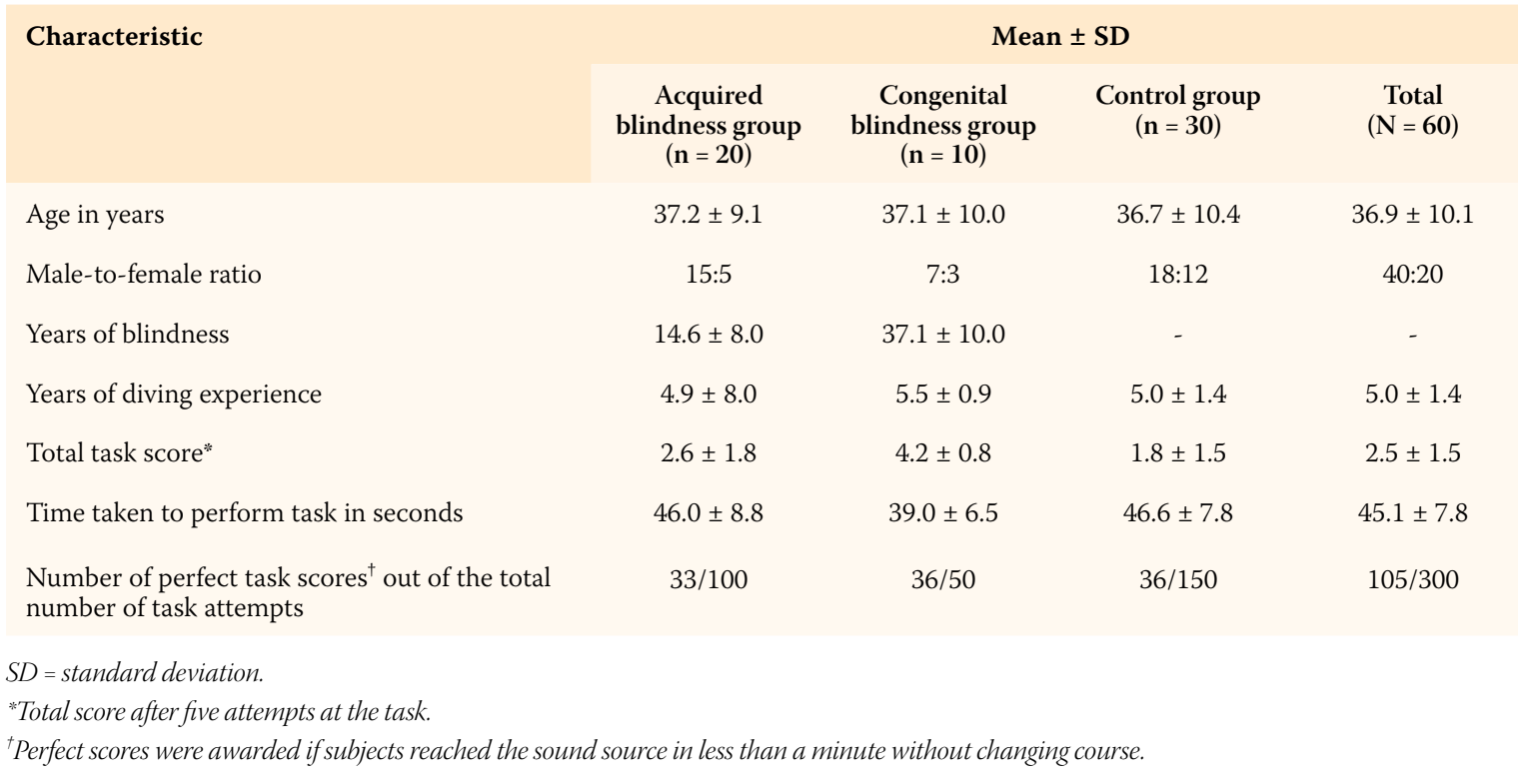




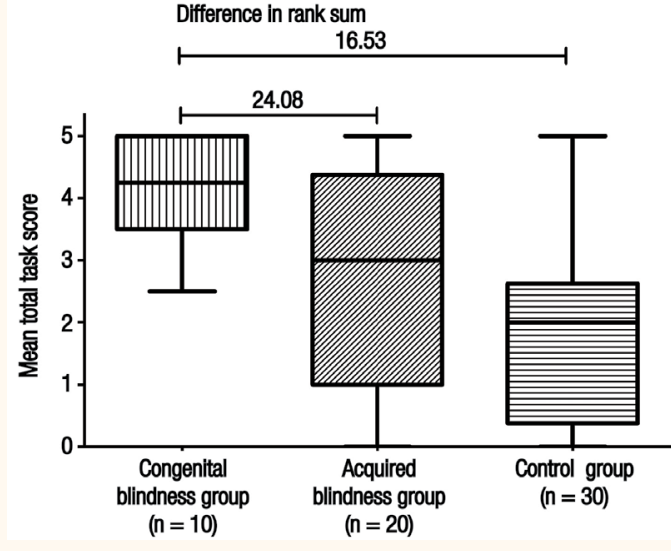

Figure 2: Bar chart showing the mean total task scores* of blind and sighted scuba divers participating in an underwater sound localisation task $(\mathrm{N}=60)$. There was a significant difference between the scores of divers who were congenitally blind in comparison to sighted subjects and those with acquired blindness $(P=0.0007){ }^{\dagger}$

"Total score after five attempts at the task.

${ }^{+}$Using Dunn's multiple comparison test.

number of years of blindness was $14.6 \pm 8.0$ and $37.1 \pm 10.0$ for the acquired blindness and congenital blindness groups, respectively [Table 1]. Among the acquired blindness group, 14 patients had retinitis pigmentosa, while three had glaucoma and one patient each had macular degeneration, a bilateral ocular injury or optic nerve atrophy. There were no significant differences in age, gender distribution or number of years of diving experience between the three groups.

There was a statistically significant difference between the total task scores of the congenital blindness and acquired blindness groups as well as between the congenital blindness and control groups $(P=0.0007)$ [Figure 2]. However, no significant difference was observed between the total scores of control subjects and those with acquired blindness. There was a significant positive association between the total sound localisation score and number of years of blindness $(P<0.0001)$ and a significant negative correlation between the time taken to execute the task and number of years of blindness $(P=0.0452)$. However, no correlations were noted between age and years of diving experience or between time taken to execute the task and years of diving experience [Table 2]. In an independent analysis of the acquired blindness group, there was a positive correlation between the number of years of blindness and the total task score $(P=0.0231)$.

\section{Discussion}

While numerous studies have investigated sound localisation among sighted and blind subjects, most have focused on comparing sound localisation in two and three dimensions or on identifying mechanisms and conditions which favour individuals who are congenitally blind. ${ }^{3,15,16}$ To the best of the authors' knowledge, the current study is the first to investigate the sound recognition capabilities of blind and sighted subjects in an underwater environment, comparing divers who were congenitally blind, those with acquired visual loss and a sighted control group whose vision was temporarily obscured. Moreover, previous research has limited sound localisation experiments among blind individuals to one horizontal plane or to a small number of potential sound source positions. ${ }^{17}$ In the current study, a high number of potential sound source locations were included on three different horizontal planes; these experimental conditions therefore more accurately reflected the real-life experience of scuba diving in an open water environment.

During auditory processing, a lag period of about 0.6 milliseconds - known as the interaural time difference (ITD) - permits location of a sound source

Table 2: Correlation matrix* between demographic and clinical characteristics and test scores among blind and sighted scuba divers participating in an underwater sound localisation task $(\mathrm{N}=60)$

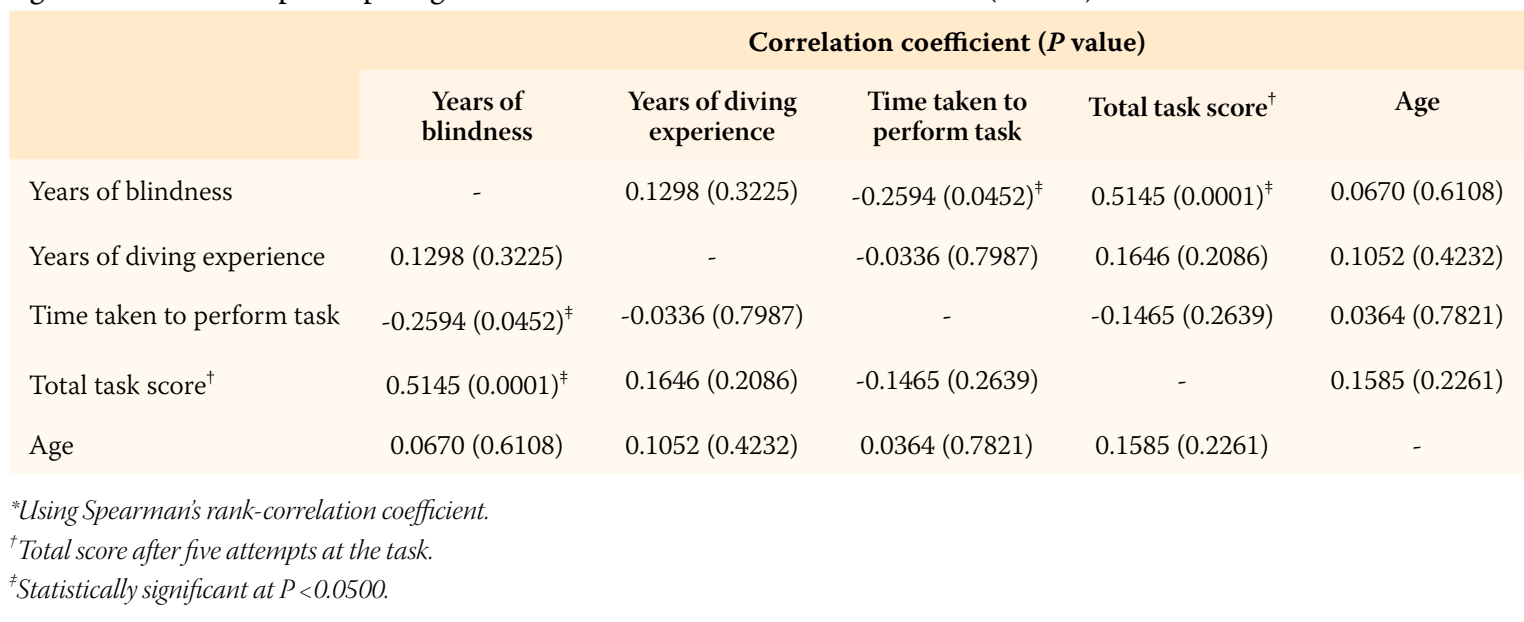


in air, but does not occur under water due to the rapid propagation of sound..$^{18}$ According to functional magnetic resonance imaging, four anatomical structures are involved during ITD: the superior olivary complex in the pons, in which loudness is analysed by the medial nuclei and spatial laterality by the lateral nuclei; the midbrain inferior colliculus, which senses duration, loudness, frequency, spatial domain, amplitude modulation and bi-aural interactions; the medial geniculate body, which determines spatial localisation and differences in interaural intensity; and the posterior parietal and frontal cortexes..$^{19,20}$

Usually, a spherical coordinate scheme is used to express the origin of a sound relative to the position or orientation of the cranium, with sounds detectable on the frontal, horizontal and median planes. When a sound arrives at the ear, the auditory signal is successively transmitted by the neuronal nuclei in the auditory pathway in the brainstem until it reaches the auditory cortex. However, underwater sounds are perceived as if they originate symmetrically in front, behind and above the subject. ${ }^{21}$ In addition, the head shadow effect (i.e. a reduction in sound amplitude due to the volume of head) is missing underwater, so the discrimination of high-frequency $(>3,000 \mathrm{~Hz})$ sound sources is difficult. ${ }^{22}$ Recently, Shupak et al. tested the use of masks with an air pocket around the ears as a method of improving underwater auditory perception; however, the authors concluded that the masks did not significantly improve underwater hearing. ${ }^{23}$

Among scuba divers, poor sound localisation is a source of concern as certain sounds may indicate imminent danger, such as an approaching motorboat. In the current study, the results of the spatial localisation tasks among the sighted controls indicated that they could only immediately identify the source of sound at a rate of $24 \%$; therefore, the task was feasible in only one out of four attempts. Hypothetically, simply guessing the source of the sound using mathematical probability would result in seven correct answers, which is much lower than the actual rate of correct localisation. Additionally, the average task execution time and rate of correction was relatively higher in the control group, with subjects making many changes in course. Divers should therefore practice sound localisation during their training so as to more accurately perceive sources of potential hazards while underwater.

As confirmed by Wightman et al., it is easier to identify the source of a sound which originates closer to the subject than those which are more remote; therefore, sound localisation tasks become much easier during execution as the subjects move closer to the source. ${ }^{24}$ In accordance with existing scientific literature, the current researchers observed that many divers started their route more medially than ideal, as if exposing one ear towards the sound so as to aid understanding of where the sound was generated. ${ }^{25}$ In addition, interaural temporal and intensity differences are reduced in water; this explains why divers have difficulty in determining from which side a sound originates. ${ }^{2}$

The results of the current study agree with those of the existing literature in demonstrating enhanced spatial sound recognition among blind subjects in comparison to sighted controls. ${ }^{3,26}$ In particular, subjects who were blind from birth spent less time reaching the sound source and more often obtained perfect results; they also changed or adjusted their direction less frequently when moving towards the source of the sound. One theory to explain these findings is that certain auditory brain areas implicated in auditory motion perception may show greater activation among people who are blind; for example, neuroimaging has revealed that the posterior parietal cortex in blind subjects is more intensely activated during tasks of immobile sound localisation and motion discrimination than in sighted controls. ${ }^{6,27-29}$ Another hypothesis focuses on compensatory plasticity in blindness, which may result in the recruitment of deafferented occipital cortical areas for the processing of auditory data; this theory is based on the strong extension and improvement of pre-existing neuronal pathways in the occipital cortex for processing auditory information. ${ }^{29,30}$ In the current study, a positive correlation was noted between number of years of blindness and task scores among subjects with acquired blindness; this finding indicates that, for two blind individuals of the same age, the individual who lost their sight earlier may have developed more effective spatial sound localisation abilities.

A major limitation of the present study was that the sounds used in the sound localisation task were not studied with a hydrophone system to determine the exact frequency and intensity of the sound upon reaching the diver. However, all subjects had normal hearing levels and they all reported hearing the rhythmic sounds very clearly. Another limitation is that, in seawater, different temperature levels can act as acoustic insulators; ${ }^{1}$ however, to counteract this, all tasks were conducted in the morning, when the sea was calm and at an average temperature of $20 \pm 2{ }^{\circ} \mathrm{C}$.

\section{Conclusion}

Subjects who were congenitally blind demonstrated superior spatial discrimination abilities in terms of locating an underwater sound source. These results 
therefore add to the growing body of evidence supporting sensory substitution or hypercompensation among individuals who are blind.

\section{CONFLICT OF INTEREST}

The authors declare no conflicts of interest.

\section{FUNDING}

No funding was received for this study.

\section{References}

1. Lurton X. An Introduction to Underwater Acoustics: Principles and applications, 2nd ed. London, UK: Springer, 2010. Pp. 13-20.

2. Hollien H. Underwater sound localization in humans. J Acoust Soc Am 1973; 53:1288-95. doi: 10.1121/1.1913466.

3. King AJ. Visual influences on auditory spatial learning. Philos Trans R Soc Lond B Biol Sci 2009; 364:331-9. doi: 10.1098/ rstb.2008.0230.

4. Kujala T, Alho K, Huotilainen M, Ilmoniemi RJ, Lehtokoski A, Leinonen A, et al. Electrophysiological evidence for crossmodal plasticity in humans with early- and late-onset blindness. Psychophysiology 1997; 34:213-16. doi: 10.1111/j.1469-8986. 1997.tb02134.x.

5. Leclerc C, Saint-Amour D, Lavoie ME, Lassonde M, Lepore F. Brain functional reorganization in early blind humans revealed by auditory event-related potentials. Neuroreport 2000; 11:545-50.

6. Weeks R, Horwitz B, Aziz-Sultan A, Tian B, Wessinger CM, Cohen LG, et al. A positron emission tomographic study of auditory localization in the congenitally blind. J Neurosci 2000; 20:2664-72.

7. Ashmead DH, Wall RS, Ebinger KA, Eaton SB, Snook-Hill MM, Yang X. Spatial hearing in children with visual disabilities. Perception 1998; 27:105-22. doi: 10.1068/p270105.

8. Lessard N, Paré M, Lepore F, Lassonde M. Early-blind human subjects localize sound sources better than sighted subjects. Nature 1998; 395:278-80. doi: 10.1038/26228.

9. Muchnik C, Efrati M, Nemeth E, Malin M, Hildesheimer M. Central auditory skills in blind and sighted subjects. Scand Audiol 1991; 20:19-23. doi: 10.3109/01050399109070785.

10. Wanet $\mathrm{MC}$, Veraart C. Processing of auditory information by the blind in spatial localization tasks. Percept Psychophys 1985; 38:91-6. doi: 10.3758/BF03202929.

11. Finocchietti S, Cappagli G, Gori M. Encoding audio motion: Spatial impairment in early blind individuals. Front Psychol 2015; 6:1357. doi: 10.3389/fpsyg.2015.01357.

12. Voss P, Lassonde M, Gougoux F, Fortin M, Guillemot JP, Lepore F. Early- and late-onset blind individuals show supranormal auditory abilities in far-space. Curr Biol 2004; 14:1734-8. doi: 10.1016/j.cub.2004.09.051.

13. Gori M, Sandini G, Martinoli C, Burr DC. Impairment of auditory spatial localization in congenitally blind human subjects. Brain 2014; 137:288-93. doi: 10.1093/brain/awt311.
14. Thinus-Blanc C, Gaunet F. Representation of space in blind persons: Vision as a spatial sense? Psychol Bull 1997; 121:20-42. doi: 10.1037/0033-2909.121.1.20.

15. Lewald J. Vertical sound localization in blind humans. Neuropsychologia 2002; 40:1868-72. doi: 10.1016/S00283932(02)00071-4.

16. Voss P, Tabry V, Zatorre RJ. Trade-off in the sound localization abilities of early blind individuals between the horizontal and vertical planes. J Neurosci 2015; 35:6051-6. doi: 10.1523/JNEU ROSCI.4544-14.2015.

17. Zwiers MP, Van Opstal AJ, Cruysberg JR. Two-dimensional sound-localization behavior of early-blind humans. Exp Brain Res 2001; 140:206-22. doi: 10.1007/s002210100800.

18. Bernstein LR. Auditory processing of interaural timing information: New insights. J Neurosci Res 2001; 66:1035-46. doi: $10.1002 /$ jnr.10103.

19. Seifritz E, Di Salle F, Esposito F, Herdener M, Neuhoff JG, Scheffler K. Enhancing BOLD response in the auditory system by neurophysiologically tuned fMRI sequence. Neuroimage 2006; 29:1013-22. doi: 10.1016/j.neuroimage.2005.08.029.

20. Engel SA, Rumelhart DE, Wandell BA, Lee AT, Glover GH, Chichilnisky EJ, et al. fMRI of human visual cortex. Nature 1994; 369:525. doi: 10.1038/369525a0.

21. Hernández-Zamora E, Poblano A. [The auditory pathway: Levels of integration of information and principal neurotransmitters]. Gac Med Mex 2014; 150:450-60.

22. Rayleigh L. Our perception of the direction of a source of sound. Nature 1876; 14:32-3. doi: 10.1038/014032a0.

23. Shupak A, Sharoni Z, Yanir Y, Keynan Y, Alfie Y, Halpern P. Underwater hearing and sound localization with and without an air interface. Otol Neurotol 2005; 26:127-30.

24. Wightman FL, Kistler DJ. Factors affecting the relative salience of sound localization cues. In: Gilkey RH, Anderson TR, Eds. Binaural and Spatial Hearing in Real and Virtual Environments. New York, USA: Lawrence Erlbaum Associates Inc., 1997. Pp. 1-24.

25. Wells MJ, Ross HE. Distortion and adaptation in underwater sound localization. Aviat Space Environ Med 1980; 51:767-74.

26. Röder B, Teder-Sälejärvi W, Sterr A, Rösler F, Hillyard SA, Neville HJ. Improved auditory spatial tuning in blind humans. Nature 1999; 400:162-6. doi: 10.1038/22106.

27. Gougoux F, Zatorre RJ, Lassonde M, Voss P, Lepore F. A functional neuroimaging study of sound localization: Visual cortex activity predicts performance in early-blind individuals. PLoS Biol 2005; 3:e27. doi: 10.1371/journal.pbio.0030027.

28. PoirierC,Collignon O,ScheiberC, RenierL, VanlierdeA, Tranduy $\mathrm{D}$, et al. Auditory motion perception activates visual motion areas in early blind subjects. Neuroimage 2006; 31:279-85. doi: 10.1016/j.neuroimage.2005.11.036.

29. Lewald J. Exceptional ability of blind humans to hear sound motion: Implications for the emergence of auditory space. Neuropsychologia 2013; 51:181-6. doi: 10.1016/j.neuropsycho logia.2012.11.017.

30. Lewald J, Meister IG, Weidemann J, Töpper R. Involvement of the superior temporal cortex and the occipital cortex in spatial hearing: Evidence from repetitive transcranial magnetic stimulation. J Cogn Neurosci 2004; 16:828-38. doi: $10.1162 / 089892904970834$ 